
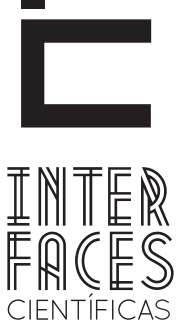

SAÚDE E AMBIENTE

ISSN IMPRESSO 2316-3313

E-ISSN 2316-3798

DOI - 10.17564/2316-3798.2017v6n1p31-42

\title{
ANÁLISE DE COMPORTAMENTOS ASSOCIADOS A DISTÚRBIOS NUTRICIONAIS EM ADOLESCENTES NORDESTINOS ${ }^{1}$
}

\section{ANALYSIS OF BEHAVIORS ASSOCIATED WITH NUTRITIONAL DISORDERS AMONG ADOLESCENTS IN BRAZIL NORTHEASTERN ANÁLISIS DE COMPORTAMIENTOS ASOCIADOS A DISTURBIOS NUTRICIONALES EN ADOLESCENTES NORDESTINOS}

Marcos Antonio Rodrigues França ${ }^{1}$

Veronica de Lourdes Sierpe Jeraldo ${ }^{3}$
Herifrania Tourinho Aragão²

\section{RESUMO}

O objetivo da pesquisa foi analisar comportamentos de adolescentes associando-os à ocorrência de distúrbios nutricionais. Foram entrevistados 694 adolescentes entre 15 e 18 anos, de sete municípios no sul de Sergipe e aplicados dois instrumentos (PAQ-C e HADS), além da avaliação antropométrica. A prevalência de baixo peso nos adolescentes foi de $4 \%$ entre os rapazes, que corresponde a $81,3 \%$ do total de adolescentes de ambos os gêneros com baixo peso e de $0,9 \%$ entre as moças, correspondendo a $18,7 \%$ de adolescentes de ambos os gêneros com esse perfil nutricional. Em relação ao sobrepeso, a prevalência entre os rapazes foi $9,0 \%$ e, entre as moças foi

1. Artigo baseado na dissertação de mestrado de Marcos Antônio Rodrigues França intitulada "Comportamentos associados ao baixo peso e ao sobrepeso
$11,1 \%$. Foram classificados como ativos fisicamente $13 \%$ dos rapazes e $9,88 \%$ das moças. Entre as moças a prevalência de ansiedade e de sintomas depressivos foi de $20,68 \%$ e $15,1 \%$ respectivamente, enquanto que nos rapazes foi de $19,14 \%$ e $12,7 \%$. A ansiedade mostrou correlação significativa com o sobrepeso entre os rapazes o mesmo não acontecendo com as moças. No entanto a correlação entre ansiedade e baixo peso se observa significativa somente entre as moças. 0 estado de depressão mostrou significativa correlação com o baixo peso e o sobrepeso entre os rapazes. Nas moças essa correlação foi significativa com o sobrepeso. Os resultados apontam esses comportamentos como variáveis

em estudantes adolescentes do Sul de Sergipe", defendida em 2008 no Programa de Pós-Graduação em Saúde e Ambiente da Universidade Tiradentes. 
de risco para o baixo peso e para o sobrepeso em ambos os gêneros e a presença deles podem ser atribuídos às pressões impostas pelas escolhas de planos futuros que 0 adolescente precisa assumir nessa fase da vida.

\section{ABSTRACT}

The objective of the research was to analyze behaviors of adolescents associating them with the occurrence of nutritional disorders. We interviewed 694 adolescents between 15 and 18 years, seven counties in southern Sergipe and two instruments (PAQ-C and HADS), besides the anthropometric assessment. The prevalence of underweight among adolescents was $4 \%$ among boys which corresponds to $81.3 \%$ of adolescents of both sexes with low weight and $0.9 \%$ among girls, accounting for $18.7 \%$ among the adolescents of both genders with this nutritional profile. Regarding overweight, the prevalence among boys was $9.0 \%$ and among girls was $11.1 \%$. In all age groups $13 \%$ of boys and $9.88 \%$ of girls were classified as physically active. Among girls the prevalence of anxiety was $20.68 \%$ and for boys was $19.14 \%$. The prevalence of depressive symptoms in the population was $15.1 \%$ for girls and $12.7 \%$ among boys. The anxiety

\section{RESUMEN}

El objetivo de la investigación fue analizar comportamientos de adolescentes asociándolos a la ocurrencia de disturbios nutricionales. Fueron entrevistados 694 adolescentes entre 15 y 18 años, de siete municipios del sur de Sergipe y aplicados dos instrumentos (PAQ-C y HADS), además de la evaluación antropométrica. La prevalencia de bajo peso en los adolescentes fue del $4 \%$ entre los varones, que corresponde al $81,3 \%$ del total de adolescentes de ambos sexos con bajo peso y del $0,9 \%$ entre las mozas, correspondiendo el $18,7 \%$ de adolescentes de ambos sexos con ese

\section{PALAVRAS-CHAVE}

Sobrepeso. Magreza. Adolescentes. was significantly correlated with overweight among boys the same not happening with the girls. However the correlation between anxiety and low birth weight observed significant only among girls. The state of depression showed significant correlation with underweight and overweight among boys. In women this correlation was significantly overweight. The results show these behaviors as risk variables for underweight and overweight in both genders and their presence can be attributed to the pressures imposed by the choices of future plans that the teenager needs to take this phase of life.

\section{KEYWORDS}

Overweight. Thinness. Adolescents.

perfil nutricional. En cuanto al sobrepeso, la prevalencia entre los varones fue del 9,0\% y, entre las jovencitas, fue el $11,1 \%$. Se clasificaron como activos físicamente el $13 \%$ de los varones y el 9,88\% de las jóvenes. Entre las muchachas la prevalencia de ansiedad y de síntomas depresivos fue el 20,68\% y 15,1\% respectivamente, mientras que en los muchachos fue el $19,14 \%$ y el $12,7 \%$. La ansiedad mostró una correlación significativa con el sobrepeso entre los varones lo mismo no sucediendo con las jóvenes. Sin embargo, la correlación entre ansiedad y bajo peso se 
observa significativa solamente entre las jóvenes. El estado de depresión mostró una significativa correlación con el bajo peso y el sobrepeso entre los varones. En las jóvenes esta correlación fue significativa con el sobrepeso. Los resultados apuntan a estos comportamientos como variables de riesgo para el bajo peso y para el sobrepeso en ambos sexos y la presencia de ellos pueden ser atribuidos a las presiones impuestas

\section{INTRODUÇ̃̃̃O}

A influência dos fatores ambientais na ocorrência de distúrbios nutricionais em adolescentes tem se mostrado importante elemento de estudos com grande potencial diagnóstico desses agravos. A maturação sexual na puberdade envolve alterações morfofuncionais neurológicas, fisiológicas, psicossociais e endócrinas. Dentre as mais evidentes, estão o acelerado aumento da estatura e da massa corporal, refletindo de maneira definitiva nas proporções corporais (FARIAS JÚNIOR et al., 2009), além das alterações do estado emocional, sendo a comportamento de ansiedade e a depressão os agravos mais evidentes (LUIZ et al., 2005).

Não existe consenso em relação à faixa etária que define a adolescência, podendo ir dos dez aos vinte anos, dos dez aos dezenove anos, ou dos doze aos dezoito anos, no entanto, alguns autores entendem não haver necessidade de rigidez nessa definição visto que essa limitação etária tem como objetivo referenciar programas e políticas públicas para a população (COUTINHO; BARROS, 2001).

Sobrepeso pode ser definido como um excesso de gordura corporal em relação à massa magra caracterizada por um índice de massa corporal percentílico (IMC) maior que $85 \%$ enquanto que baixo peso, definido pelo IMC percentílico menor que 5\% (BRASIL, 2006), é a deficiência ou falta de nutrientes - proteínas, carboidratos, vitaminas e aminoácidos - essenciais para o bom desenvolvimento do indivíduo. Segundo Lourenço e outros autores (2011), é possível diagnosticar distúrbios nutricionais, tais como magreza e excesso de peso por meio de critérios antro- por las elecciones de planes futuros que el adolescente necesita asumir en esa fase de la vida.

\section{PALABRAS CLAVE}

Sobrepeso, Delgadez, Adolescentes. pométricos, além de auxiliar o profissional de saúde a promover ações de saúde na conduta nutricional.

No tocante aos distúrbios de composição corporal, o sedentarismo ocupa posição de destaque entre os comportamentos relacionados ao sobrepeso e baixo peso (REBELLO; COSTA, 2007). Como elemento agravante desse processo nas populações humanas, a urbanização vem privilegiando o uso dos espaços ociosos para objetivos econômicos e quando existem áreas voltadas para o lazer, estas são empreendimentos particulares que excluem a maior parte da população que não pode usufruí-las da forma e momento que the convier.

Esses indivíduos, expostos ao comportamento sedentário têm negada a possibilidade da prática da atividade física, que é entendida como um conjunto de movimentos corpóreos produzidos pelos músculos esqueléticos que resultam em gasto energético. A atividade física caracteriza-se pela multidimensionalidade das ações corporais voluntárias que envolvem movimentos laborais, de lazer e desportivas (MENDONÇA; DOS ANJOS, 2004) e que possui relação inversa com as doenças crônico-degenerativas (WEINECK, 2003), além de ser base sólida para a redução da prevalência do sedentarismo na idade adulta, contribuindo desta forma para um estilo de vida saudável e uma melhor qualidade de vida (BRASIL, 2006).

As alterações do estado psicológico, da mesma forma que o sedentarismo e situação socioeconômica, caracterizam-se como importantes fatores de risco para os distúrbios da composição corporal nos 
adolescentes. A exposição a elevadas quantidades de informações sobre modelos e padrões corporais, reforça indícios de que este comportamento pode ser desencadeador de manifestações psicológicas, como depressão e comportamento de ansiedade (MARANHÃO NETO; MARTINS DE MIRANDA, 2003).

Esses arquétipos impostos pelo meio sociais, que acabam por perpetuar a insatisfação com o peso corporal e é reflexo de uma cultura de valorização à magreza ao mesmo tempo em que discriminam e excluem os obesos, podem refletir de forma negativa na ingestão quanti-qualitativa de alimentos; especialmente entre as mulheres que dispensam mais atenção aos padrões de beleza, submetendo-se a dietas restritivas e de baixo valor energético (ALMEIDA; LOUREIRO; SANTOS, 2002).

Segundo Vitolo e outros autores (2007), após avaliar diferentes metodologias para classificação de excesso de peso em adolescentes brasileiros, é indispensável a investigação de valores de referência baseados em estudos distribuídos homogeneamente no território brasileiro, pois a aplicação dos dados, tanto no âmbito clínico como no epidemiológico, terá methor representatividade. Desta forma, o presente trabalho objetivou analisar comportamentos assumidos por adolescentes de escolas públicas do sul de Sergipe, associando-os à ocorrência de baixo peso e de sobrepeso.

\section{MATERIAL E MÉTODOS}

Este estudo caracteriza-se por ser epidemiológico transversal e descritivo. A área de estudo foi delimitada a partir da denominação estabelecida pela Secretaria de Estado de Educação e Cultura de Sergipe que agrupa os municípios por Delegacias Regionais de Educação (DRE). A DRE escolhida, denominada DRE 02, situa-se no sul do estado e abrange sete municípios: Lagarto, Tobias Barreto, Simão Dias, Riachão do Dantas, Poço Verde, Salgado e Boquim. A região possui nível de alfabetização superior a 89\% com 8.753 adolescentes matriculados na faixa etária contemplada.
Utilizou-se como fator de homogeneização dos municípios selecionados, o índice de desenvolvimento humano (IDH), indicador baseado na combinação de três dimensões - longevidade, educação e renda - sendo assim classificados como de médio desenvolvimento humano - 0,500 a 0,800 (BRASIL, 2006). A população em estudo foi identificada como todos os alunos matriculados nas escolas da rede estadual da região contemplada (15 a 18 anos) durante o ano de 2008. A amostra, composta por 694 indivíduos, foi caracterizada como aleatória e proporcional por município, uniforme e estratificada por gênero e idade, intencional por turma e turno de aulas.

A coleta de dados foi realizada em dois momentos, por meio de dois instrumentos: um questionário, contendo informações acerca da situação sócio econômica dos indivíduos, atividades físicas cotidianas - PAQ-C e comportamento de ansiedade e depressão - HADS e a avaliação antropométrica, com a mensuração da estatura e massa corporal, realizada naqueles indivíduos que devolveram o questionário corretamente preenchido.

$\mathrm{Na}$ análise estatística dos dados, determinaram-se as medidas absolutas e de prevalências, medidas de tendência central e de dispersão dos comportamentos e das mensurações antropométricas. As análises bivariadas de correlação linear, associação qui-quadrado, teste de correlação de Spearman e Odds ratio, com significância $\leq 0,05$, foram empregadas para analisar possíveis relações entre as variáveis do estudo. Estas análises foram realizadas com uso do programa SPSS, versão 15.0. 0 estudo foi aprovado pelo Comitê de Ética em Pesquisa da Universidade Tiradentes (UNIT), sob protocolo $\mathrm{n}^{0} 110307$.

\section{RESULTADOS}

A Tabela 1 mostra medidas de tendência central e comparação de médias entre os gêneros, na qual se observaram os valores médios mais elevados nos rapazes com relação à massa corporal, estatura e nível 
de atividade física com exceção do IMC - razão entre o quadrado da altura e a massa corporal.

Tabela 1 - Avaliação antropométrica e do nível de atividade física, segundo o PAQ-C, entre estudantes adolescentes do sul do estado de Sergipe, 2008

\begin{tabular}{lcccc}
\hline & \multicolumn{2}{c}{ Rapazes } & \multicolumn{2}{c}{ Moças } \\
\cline { 2 - 5 } & Média & Dp & Média & Dp \\
\hline IMC* & 20,77 & 3,30 & 21,48 & 2,34 \\
\hline $\begin{array}{l}\text { Massa corporal } \\
\text { (Quilos)* }\end{array}$ & 56,34 & 8,72 & 53,10 & 6,04 \\
\hline Estatura (Metros)* & 1,64 & 0,07 & 1,57 & 0,63 \\
\hline $\begin{array}{l}\text { Nível de atividade } \\
\text { física } \\
\text { (PAQ-C Score 1 a 5) }\end{array}$ & 2,64 & 0,61 & 2,51 & 0,50 \\
\hline
\end{tabular}

${ }^{*} \mathrm{p} \leq 0,05$ / dp - desvio padrão

Fonte: Dados da pesquisa.

Independente do gênero, a prevalência global para baixo peso foi de $3,39 \%$. Ao levar-se em consideração o gênero (FIGURA 1), a prevalência de baixo peso entre os rapazes foi ligeiramente mais elevada com $4,01 \%(n=13)$ versus $2,78 \%(n=9)$ entre as moças sem, no entanto, ser essa diferença considerada significativa $(p \geq 0,05)$. Quanto ao sobrepeso, a prevalência global foi de 10,03\%. Enquanto entre os rapazes $8,95 \%(n=29)$ estavam acima do peso, este evento ocorreu em $11,11 \%(n=36)$ das moças.
Figura 1 - Distribuição percentual das prevalências de baixo peso, eutrofia e sobrepeso nos estudantes adolescentes do sul de Sergipe, 2008

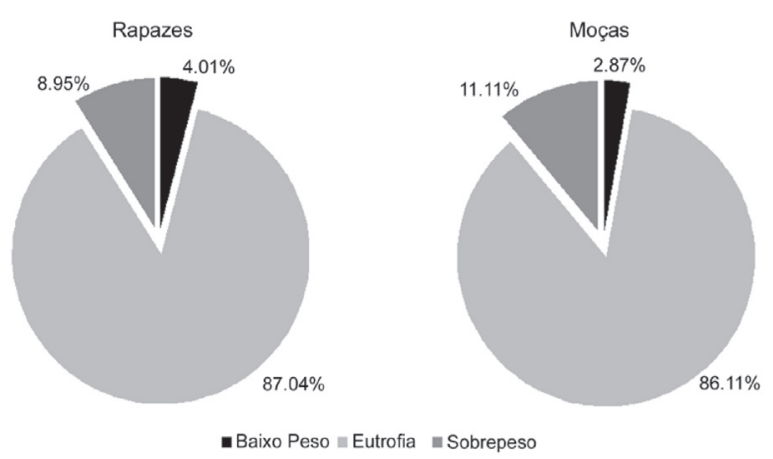

Fonte: Dados da pesquisa.

Com exceção da análise entre estatura e IMC nos rapazes com 16 anos $(p=0,06)$ houve significativa correlação $(p \leq 0,05)$ entre as variáveis antropométricas em todas as idades e em ambos os gêneros (TABELA 2). No entanto, exceto na análise do IMC correlacionado à massa corporal, como mostra a Figura $2 \mathrm{~A}$, indicando "forte" correlação com elevados valores de " $r$ " $(>0,600)$, todas as correlações foram classificadas como "regular" (FIGURAS 2B e 2C).

$\mathrm{Na}$ Figura 2B, pode-se observar uma correlação linear negativa e significativa ( $p \leq 0,05)$, porém, muito baixa entre a estatura e o IMC dos adolescentes sergipanos (-0,396 rapazes e $-0,324$ moças), ao contrário da figura $2 \mathrm{C}$, que apresenta uma tendência de correlação positiva (0,771 rapazes e 0,692 moças).

Figura 2 - Correlação linear entre (A) massa corporal e IMC, (B) estatura e IMC e (C) estatura e massa corporal dos estudantes adolescentes do sul de Sergipe, 2008
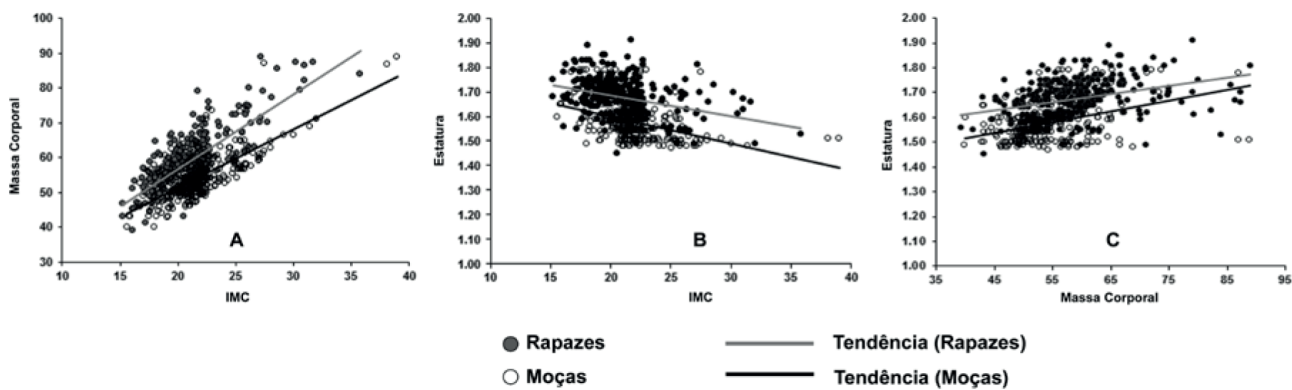

Fonte: Dados da pesquisa. 
Tabela 2 - Coeficiente de Correlação de Spearman entre as variáveis IMC, massa corporal e estatura dos estudantes adolescentes do sul de Sergipe, 2008

\begin{tabular}{|c|c|c|c|c|c|}
\hline & 15 anos & 16 anos & 17 anos & 18 anos & Total \\
\hline \multicolumn{6}{|l|}{ RAPAZES } \\
\hline IMC x Massa Corporal & $0,820 *$ & $0,799 *$ & $0,702^{*}$ & $0,767^{*}$ & $0,771^{*}$ \\
\hline Massa Corporal x Estatura & $0,296 *$ & $0,419 *$ & $0,259 *$ & $0,301^{*}$ & $0,346^{*}$ \\
\hline Estatura x IMC & $-0,297^{*}$ & $-0,207$ & $-0,499 *$ & $-0,037^{*}$ & $-0,324^{*}$ \\
\hline \multicolumn{6}{|l|}{ MOÇAS } \\
\hline IMC x Massa Corporal & $0,707^{*}$ & $0,593^{*}$ & $0,682^{*}$ & $0,761^{*}$ & $0,692^{*}$ \\
\hline Massa Corporal x Estatura & $0,392^{*}$ & $0,368^{*}$ & $0,277^{*}$ & $0,436^{*}$ & $0,382^{*}$ \\
\hline Estatura x IMC & $-0,364$ * & $-0,528$ * & $-0,508^{\star}$ & $-0,246^{*}$ & $-0,396^{*}$ \\
\hline
\end{tabular}

* Correlação significativa $(p \leq 0,05)$

Fonte: Dados da pesquisa.

Com relação ao nível de atividade física, em todas as faixas etárias estudadas, menos de um terço (13\%; $n=42)$ dos rapazes e $9,88 \%(n=32)$ das moças foram classificados como suficientemente ativos fisicamente. Independente do gênero, a grande maioria apresentou níveis insuficientes de atividade física. Esse comportamento sedentário apresentou-se de forma mais contundente nas moças $(90,1 \%$; $n=292)$ do que nos rapazes $(87,04 \% ; n=282)$. Exceto aos 17 anos, em todas as demais idades os rapazes estudados mostraram um comportamento fisicamente mais ativo.

A prevalência global de sintomas de comportamento de ansiedade na população estudada foi de $19,91 \%$. Entre as moças a prevalência foi de $20,68 \%$ com a idade de 15 anos, apresentando os maiores valores percentuais (35,82\%). Entre os rapazes a prevalência foi de $19,14 \%$ e a idade de 18 anos a de maior prevalência com $32,26 \%$. A maior ocorrência de sintomas de comportamento de ansiedade acontece aos 18 anos entre os rapazes (25\%) e 15 anos entre as moças (30\%).

0 comportamento de ansiedade, pelo critério da HADS, mostrou correlação significativa $(p \leq 0,05)$ com o sobrepeso entre os rapazes, não acontecendo o mesmo com as moças. No entanto, o quadro se inverte na correlação entre comportamento de ansiedade e baixo peso onde se observa correlação significativa somente entre as moças $(p \leq 0,05)$.
A prevalência global de sintomas depressivos na população de adolescentes sergipanos foi de $13,9 \%$, apontando registros de $15,1 \%$ no gênero feminino e de $12,7 \%$, no gênero masculino. Apesar do expressar comportamentos distintos entre os gêneros, os valores de prevalência de sintomas de depressão mostram-se mais elevados e similares na idade de 18 anos em ambos os gêneros sem, no entanto, mostrar correlação significativa $(p \leq 0,05)$ entre essa idade e os distúrbios nutricionais de baixo peso e sobrepeso (TABELA 3).

A Tabela 3 mostra, ainda, que estado de depressão, classificado pela HADS, mostrou significativa correlação com o baixo peso e o sobrepeso entre os rapazes. Nas moças essa correlação foi significativa com o sobrepeso. A razão de chance (Odds Ratio) para ocorrência de baixo peso e sobrepeso na presença de comportamento de ansiedade e de depressão nos adolescentes estudados pode ser observada também na Tabela 3.

0 risco dos rapazes sergipanos com distúrbios nutricionais (baixo peso e sobrepeso) foi em média 1,67 vezes mais elevado quando estes adolescentes apresentavam comportamentos de depressão (OR=4,31 e 4,87 ). As moças, no entanto, apresentaram maior risco de baixo peso (OR=7,67, TABELA 3) quando apresentavam comportamento de ansiedade. 
Tabela 3 - Coeficiente de Correlação de Spearman $\left(r_{s}\right)$ e razão de chance (Odds Ratio) de ocorrência de baixo peso e sobrepeso entre adolescentes do sul de Sergipe com sintomas de comportamento de ansiedade e/ou depressão, por gênero no sul de Sergipe, 2008.

\begin{tabular}{|c|c|c|c|c|c|c|c|c|}
\hline & \multicolumn{4}{|c|}{ RAPAZES } & \multicolumn{4}{|c|}{ MOÇAS } \\
\hline & \multicolumn{2}{|c|}{ Baixo peso } & \multicolumn{2}{|c|}{ Sobrepeso } & \multicolumn{2}{|c|}{ Baixo peso } & \multicolumn{2}{|c|}{ Sobrepeso } \\
\hline & Correlação & $\begin{array}{c}\text { OR } \\
(\mathrm{IC} 95 \%)\end{array}$ & Correlação & $\begin{array}{c}\text { OR } \\
(\mathrm{IC} 95 \%)\end{array}$ & Correlação & $\begin{array}{c}\text { OR } \\
(\mathrm{IC} 95 \%)\end{array}$ & Correlação & $\begin{array}{c}\text { OR } \\
(\mathrm{IC} 95 \%)\end{array}$ \\
\hline ANSIEDADE & $\begin{array}{l}r_{s}=0,100 \\
p=0,07\end{array}$ & $\begin{array}{c}2,64 \\
(0,89-7,79)\end{array}$ & $\begin{array}{l}r_{s}=0,177 \\
p=0,00\end{array}$ & $\begin{array}{c}2,98 \\
(1,50-5,91)\end{array}$ & $\begin{array}{l}r_{s}=0,192 \\
p=0,00\end{array}$ & $\begin{array}{c}7,67 \\
(1,97-29,87)\end{array}$ & $\begin{array}{l}r_{s}=0,086 \\
p=0,12\end{array}$ & $\begin{array}{c}1,68 \\
(0,87-3,92)\end{array}$ \\
\hline DEPRESSÃO & $\begin{array}{l}r_{s}=0,159 \\
p=0,00\end{array}$ & $\begin{array}{c}4,31 \\
(1,48-12,55)\end{array}$ & $\begin{array}{l}r_{s}=0,271 \\
p=0,00\end{array}$ & $\begin{array}{c}4,87 \\
(2,51-9,45)\end{array}$ & $\begin{array}{l}r_{s}=0,086 \\
p=0,12\end{array}$ & $\begin{array}{c}2,80 \\
(0,72-10,84)\end{array}$ & $\begin{array}{l}r_{s}=0,152 \\
p=0,00\end{array}$ & $\begin{array}{c}2,89 \\
(1,31-6,35)\end{array}$ \\
\hline
\end{tabular}

(OR - Odds Ratio; IC95\% - Intervalo de confiança)

Fonte: Dados da pesquisa.

\section{DISCUSSÃO}

Estima-se que mais de um bilhão de pessoas no mundo são afetadas pelo sobrepeso com cerca de um terço dessa população considerada obesa (BRASIL, 2006). No Brasil, estima-se que $20 \%$ das crianças sejam obesas e que cerca de $30 \%$ da população adulta apresentem algum grau de excesso de peso.

Os padrões antropométricos e nível de atividade física distintos entre os sexos dos adolescentes sergipanos (TABELA 1) podem estar relacionados ao fato de que na mulher a massa muscular diminui em relação ao peso corporal total, devido a um aumento acelerado do tecido adiposo, enquanto no homem devido à ação dos hormônios masculinos ocorre um aumento da massa livre de gordura e consequente aumento de força muscular (FARIAS JÚNIOR et al., 2009).

Os distúrbios e carências nutricionais estão hoje elencados como um dos maiores problemas enfrentados por sociedades em desenvolvimento, não só pela sua elevada ocorrência, mas também, enquanto fator de risco para o acometimento de inúmeras doenças decorrentes desse problema (SALVE, 2006). Os valores de magreza nos adolescentes sergipanos, no entanto, são bem inferiores aos mapeados em um amplo estudo sobre estado nutricional da população brasileira (BRASIL, 2006), que observou nos adultos jovens (20 a 24 anos) uma prevalência maior de déficit de peso (12,2\%). Esse fato pode ser consequência da implantação e popularização de políticas e programas de saúde pública voltados para o controle de enfermidades que têm o baixo peso e a desnutrição como evidente fator de risco, a exemplo do "Bolsa Família", Estratégia de Saúde da Família (ESF), além da melhoria da eficiência na distribuição da merenda escolar.

Estado nutricional similar no sexo masculino foi encontrado em estudo realizado por Balaban e Silva (2001), com uma prevalência de 6,20\% para o sobrepeso nos rapazes. 0 estado nutricional das moças, por sua vez, parece não ser diferente daquele relatado por levantamento do Ministério do Desenvolvimento Social e Combate a Fome, em 2006 - 13,40\% (BRASIL, 2006).

As prevalências equitativas de baixo peso entre as moças e rapazes sergipanos $(p \geq 0,05)$ é um cenário semelhante ao encontrado por Dutra e outros autores (2006) na região sul do Brasil. No entanto, é importante ressaltar que o modelo de referência de crescimento e desenvolvimento atualmente utilizado no Brasil, não reflete a nossa realidade devido às dimensões geográficas e a variabilidade de influências ambientais e sociais observadas nas diferentes regiões (GUEDES et al., 2013).

Atualmente, independente da caracterização socioeconômica, não mais se estabelece relação entre desenvolvimento e obesidade (ADES; KERBAUY, 2002), prova disso foi o fenômeno epidemiológico da 
transição nutricional, evidenciado pelos inquéritos nacionais realizados em 1974/1975 e 1989 (MONTEIRO et al., 1993) que mostrou a tendência de alteração no perfil nutricional e na composição corporal de crianças, diminuindo a prevalência de desnutrição e aumentando a prevalência de sobrepeso e obesidade. As correlações apresentadas neste estudo indicam que esse fenômeno se encontra em estágio de desenvolvimento na população juvenil sergipana. A correlação linear negativa, porém, muito baixa entre a estatura e o IMC, além da correlação positiva forte entre massa corporal e IMC evidencia a maior importância desta última no índice de massa corporal dos adolescentes sergipanos (CALLEGARI-JACQUES, 2011).

Ao se levar em consideração a afirmação de Araújo e Oliveira (2008) de que a massa corporal realmente exerce influência maior do que a estatura na composição do IMC fica evidente na população aqui estudada, um desequilíbrio na correlação entre estatura e massa corporal, evidenciando que mesmo sob a influência do estirão de crescimento que ocorre na adolescência, a estatura não consegue suplantar o incremento do ganho de peso. Esse fato pode dever-se a comportamentos sedentários recém-adquiridos por este grupo populacional, como maior acesso à compra de veículos ciclomotores, abandono ou destruição de áreas destinadas ao lazer e disseminação da internet como meio de entretenimento.

A obesidade contribui para a diminuição da expectativa de vida, especialmente quando a exposição é por tempo prolongado, desde a infância ou adolescência, podendo contribuir para um aumento da mortalidade por doenças cardiovasculares, em especial no gênero masculino (CAVALCANTI et al., 2010). Estudo epidemiológico sobre as condições de saúde infantil tem mostrado que adolescentes obesos são mais propensos a permanecerem obesos na vida adulta (CARDOSO et al., 2010).

Com o avanço tecnológico, o homem reduziu à quase nulidade o seu trabalho físico e apesar do aumento da expectativa de vida nos países em desenvolvimento (BRASIL, 2006), os casos de mortes no mundo que são atribuídas à inatividade física, ultrapassa os dois milhões, na medida em que esta se posiciona como principal comportamento de risco para as doenças crônicas não transmissíveis.

O comportamento sedentário dos adolescentes sergipanos é reforçado por pesquisadores que afirmam que os níveis de atividade física tendem a diminuir com a adolescência e que os meninos são mais ativos e melhores condicionados fisicamente que as meninas (GUERRA; FARIAS JÚNIOR; FLORINDO, 2016). Souza e Duarte (2005) relatam que, independente do gênero, os níveis de atividade física diminuem com a idade. Na região sul sergipana, o comportamento descrito acima pode estar relacionado à baixa qualificação de grande parte dos professores de educação física que adotam como principais conteúdos de ementas as atividades competitivas, na maioria das vezes excludentes e repetitivas, não privilegiando na sua prática diária a aquisição de um comportamento crítico e proativo ante a atividade física enquanto comportamento preventivo de promoção da saúde.

Outra justificativa poderia estar relacionada à melhoria nos meios de transporte, levando a substituição dos deslocamentos a pé e de bicicleta por deslocamentos via ciclomotores, bastante utilizados por pessoas de todas as idades e para os mais diversos afazeres, além da internet que tem mostrado ser a principal opção de lazer dos adolescentes que dispensam grande parte do seu tempo ocioso em frente a computadores, tanto no ambiente domiciliar, quanto em lan houses.

$\mathrm{Na}$ medida em que a adolescência se caracteriza como uma fase de aquisição e sedimentação de comportamentos (COUTINHO; BARROS, 2001) e uma vez que os adolescentes estudados mostraram baixos níveis de atividade física ( $\approx 87$ a $90 \%$ ), existe uma maior perspectiva de, na idade adulta perpetuarem essas condutas, ficando mais expostos aos agravos que têm no sedentarismo um fator de risco (SOUZA et al., 2007).

A ansiedade e a depressão têm sido cada vez mais relatadas em estudos que têm como tema os distúrbios da composição corporal; as manifestações de ansiedade e depressão quando relacionadas aos distúrbios nutricionais, normalmente são frutos da influ- 
ência do meio social que estabelece padrões corporais, interferindo no modo como esse indivíduo percebe 0 próprio corpo (ALMEIDA; LOUREIRO; SANTOS, 2002).

Apesar da diversidade de instrumentos utilizados em outros trabalhos na avaliação dos sintomas de ansiedade e depressão, as prevalências são bastante semelhantes. Jatobá e Bastos (2007) realizaram levantamento em 10.414 alunos de escolas do ensino médio da rede pública e privada em Recife, utilizando o questionário de sintomas de ansiedade e encontrou prevalência global $(19,9 \%)$, o mesmo valor ao do presente estudo (19,91\%), alcançando níveis de 61,9\% dos casos no gênero feminino.

A ocorrência de ansiedade entre as moças e rapazes sergipanos coincide com a desaceleração do crescimento e desenvolvimento físico de cada gênero e pode estar relacionado com o processo de aceitação da imagem corporal recém-adquirida (CONTI; GAMBARDELLA; FRUTUOSO, 2005). Padrão equivalente foi observado na prevalência global de sintomas depressivos entre os adolescentes sergipanos, quando comparada com registros obtidos na região sul do Brasil (SUKIENNIKet al., 2000).

Os sintomas depressivos dos adolescentes sergipanos não apresentam correlação significativa $(p \leq 0,05)$ entre essa idade e os distúrbios nutricionais de baixo peso e sobrepeso, o que revela consonância com o estudo realizado por Kakeshita e Almeida (2006) em um grupo de jovens de São Paulo o qual indica que paralelamente ao término do processo de crescimento e desenvolvimento, diversos fatores exógenos aos distúrbios nutricionais influenciam na aquisição dos comportamentos de ansiedade e depressão. As correlações significativas observadas entre o estado de depressão dos jovens sergipanos e o baixo peso/ sobrepeso podem configurar-se em, aparentemente, um indicador da preocupação exacerbada das adolescentes em relação ao controle de massa corporal.

A razão de chance (OR) de ansiedade e depressão nos adolescentes sergipanos aponta esses comportamentos como variáveis de risco para o baixo peso e para o sobrepeso em ambos os gêneros, atribuindo a presença deles às pressões impostas pela série de es- colhas de planos futuros que o adolescente, com personalidade ainda em formação, precisa assumir nessa fase da vida, como proximidade com o vestibular, escolhas e expectativas profissionais (LUIZ et al., 2005).

\section{CONCLUSÃO}

Estudos sobre a ocorrência de sobrepeso e baixo peso e suas relações com fatores psicossociais, ambientais e fisiológicos, devem ser realizados em diversas regiões geográficas, apresentando perfis socioeconômicos diferentes, de forma a possibilitar a geração de conhecimento mais adequado a subsidiar orientações e políticas públicas a nível local, regional, nacional ou, mesmo, mundial. Esses estudos, fundamentais para qualquer tipo de planejamento público que vise à melhoria da qualidade de vida da população, necessariamente devem ser realizados por equipes interdisciplinares, sob o risco de não poder ser possível compreender os aspectos multi-contextuais que envolvem os fatores determinantes dos distúrbios nutricionais.

Em vista do exposto, salienta-se a relevância dos resultados apresentados nesse trabalho no planejamento de estratégias para a veiculação de informações para a população em geral no sentido da redução da ocorrência e consequentemente da incidência de distúrbios nutricionais, incorporando um estio de vida sadio, além de desenvolver uma cultura de bem-estar aliado a elevados níveis de qualidade de vida.

\section{REFERÊNCIAS}

ADES, L.; KERBAUY, R.R. Obesidade: realidade e indagações. São Paulo: Instituto de Psicologia da Universidade de São Paulo, 2002.

ALMEIDA, G.A.N.; LOUREIRO, S.R.; SANTOS, J.E. A imagem corporal de mulheres morbidamente obesas 
avaliadas através do desenho da figura humana.

Psicol. Refl. Crít., v.15, n.2, p.283-292, 2002.

ARAÚJO, S.S.; OLIVEIRA, A.C.C. Aptidão física em escolares de Aracaju. Rev. Bras. Cineantropom.

Desempenho Hum., v.10, n.3, p.271-276, 2008.

BALABAN, G.; SILVA, G.A.P. Prevalência de sobrepeso e obesidade em crianças e adolescentes de uma escola da rede privada de Recife. J Pediatr., v.77, n.2, p.96-100, 2002.

BRASIL. Ministério da Saúde. Secretaria de Atenção à Saúde. Cadernos de Atenção Básica. Obesidade. Brasília: Ministério da Saúde, 2006.

\section{CALLEGARI-JACQUES, S.M. Bioestatística}

princípios e aplicações. Porto Alegre: Artmed, 2011.

CARDOSO, C.B.M.A. et al. Obesidade na adolescência: reflexões e abordagem. Adolescência \& Saúde, v.7, n.1, p.12-18, 2010.

CAVALCANTI, C.L. et al. Envelhecimento e Obesidade: um Grande Desafio no Século XXI. Rev.

Bras. Cien. Saúde, v.14, n.2, p.87-92, 2010.

CONTI, M.A.; GAMBARDELLA, A.M.D.; FRUTUOSO, M.F.P. Insatisfação com a imagem corporal em adolescentes e sua relação com a maturação sexual.

Rev. Bras. Crescimento Desenvolv. Hum., v.15, n.2, p.36-44, 2005.

COUTINHO, M.F.G.; BARROS, R.R. Adolescência: uma abordagem prática. São Paulo: Atheneu, 2001.

DUTRA, L.C.; ARAÚJO, C.L.; BERTOLDI, A.D.

Prevalência de sobrepeso em adolescentes: um estudo de base populacional em uma cidade no sul do Brasil.

Cad. Saúde Pública, v.22, n.1, p.151-162, 2006.

FARIAS JÚNIOR, J.C. et al. Comportamentos de risco à saúde em adolescentes no sul do Brasil: prevalência e fatores associados. Rev. Panam. Salud Pública, v.25, n.4, p.344-352, 2009.

GUEDES, D.P. et al. Baixo peso corporal/magreza, sobrepeso e obesidade de crianças e adolescentes de uma região brasileira de baixo desenvolvimento econômico. Rev. Paul. Pediatr., v.31, n.4, p.437443, 2013.

GUERRA, P.H.; FARIAS JÚNIOR, J.C.; FLORINDO, A.A. Comportamento sedentário em crianças e adolescentes brasileiros: revisão sistemática. Rev. Saúde Pública, v.50, p.1-15, 2016.

JATOBÁ, J.A.V.N.; BASTOS, O. Depressão e ansiedade em adolescentes de escolas públicas e privadas. J.

Bras. Psiquiatr., v.56, n.3, p.171-179, 2007.

KAKESHITA, I.S.; ALMEIDA, S.S. Relação entre índice de massa corporal e a percepção da auto-imagem em universitários. Rev. Saúde Pública, v.40, n.3, p.497504, 2006.

LOURENÇO, A.M.; TAQUETTE, S.R.; HASSELMANN, M.H. Avaliação nutricional: antropometria e conduta nutricional na adolescência. Adolesc. Saude, v.8, n.1, p.51-58, 2011.

LUIZ, A.M.A.G. et al. Depressão, ansiedade e competência social em crianças obesas. Estud.

Psicol., v.10, n.1, p.35-39, 2005.

MARANHÃO NETO, G.A.; MARTINS DE MIRANDA, C.J. Detecção do risco de sobrepeso em servidoras universitárias: um estudo exploratório. Rev. Bras.

Fisiol. Exerc., v.2, p.166-171, 2003.

MENDONÇA, C.A.; DOS ANJOS, L.A. Aspectos das práticas alimentares e da atividade física como determinantes do crescimento do sobrepeso/ obesidade no Brasil. Cad. Saúde Pública, v.20, n.3, p.698-709, 2004. 
MONTEIRO, C.A. et al. ENDEF e PNSN: para onde caminha o crescimento físico da criança brasileira? Cad. Saúde Pública, v.9, suppl.1, p.85-95, 1993.

REBELLO, W.J.; COSTA, R.F. Estudo comparativo de sobrepeso e obesidade em adolescentes em duas escolas da cidade de Curitiba. Rev. Bras. Prescr. Fisiol. Exerc., v.1, n.1, p.55-60, 2007.

SALVE, M.G.C. Obesidade e peso corporal: riscos e consequências. Mov. Percep., v.6, n.8, p.29-48, 2006.

SOUZA, D.P. et al. Etiologia da obesidade em crianças e adolescentes. Rev. Bras. Nutr. Clín., v.22, n.10, p.72-76, 2007.
SOUZA, G.S.; DUARTE, M.F. Estágios de mudança de comportamento relacionados à atividade física em adolescentes. Rev. Bras. Med. Esporte, v.11, n.2, p.104-108, 2005.

SUKIENNIK, P.B. et al. Implicações da depressão e do risco de suicídio na escola durante a adolescência. Adolesc. Latinoam., v.2, n.1, p.36-44, 2000.

VITOLO, M.R. et al. Avaliação de duas classificações para excesso de peso em adolescentes brasileiros.

Rev. Saúde Pública, v.41, n.4, p.653-656, 2007.

WEINECK, J. Atividade física e esporte: para quê? São Paulo: Manole, 2003. 
Recebido em: 23 de Maio de 2017

Avaliado em: 24 de Maio de 2017

Aceito em : 7 de Junho de 2017
1. Educador Físico; Mestre em Saúde e Ambiente pela Universidade Tiradentes; Professor do Instituto Federal de Educação, Ciência e Tecnologia de Sergipe - IFS. Email: marcosfranca@cefetse.edu.br

2. Enfermeira; Programa de Pós-Graduação em Saúde e Ambiente, Universidade Tiradentes - UNIT. Email: fanyaragao.89@gmail.com

3. Doutora em Ciências pela Universidade de São Paulo; Laboratório de Doenças Infecciosas e Parasitárias - Instituto de Tecnologia e Pesquisa. Email: veronica_sierpe@hotmail.com 